\title{
Low prevalence of HIV infection, and knowledge, attitude and practice on HIV/AIDS among high school students in Gondar, Northwest Ethiopia
}

\author{
Gashaw Andargie ${ }^{1}$, Afework Kassu² ${ }^{2}$ Feleke Moges ${ }^{2}$, Yigzaw Kebede ${ }^{3}$, Molla Gedefaw ${ }^{3}$, Fisseha Wale ${ }^{4}$, Atnaf Alem , \\ Berhanu Andualem ${ }^{5}$ Solomon Adungna ${ }^{6}$
}

\begin{abstract}
HIV/AIDS is a major public health problem in Ethiopia. Therefore, a school based cross-sectional study was conducted in Gondar; Northwest Ethiopia to determine the seroprevalence of HIV infection and to assess Knowledge, attitude and practice related to HIV/AIDS. A total of 565 students were included in the study. The seroprevalence of HIV infection was $1.1 \%$. Sexual contact with commercial sex worker or non-regular partner was reported by $16.7 \%$ of the students. Only 58.5\% of those who practice sex used condoms. History of sexually transmitted diseases was reported by $10.7 \%$ of the sexually active students. The majority (96.6\%) reported unprotected sex, unsafe blood transfusion, contaminated needles and mother to child transmissions as common ways of HIV transmission. Abstinence, faithfulness to one's partner and use of condom as means to prevent transmission of HIV was responded by $84.1 \%, 60.4 \%$ and $41.8 \%$ of the students, respectively. Over $82 \%$ demanded screening for HIV as a precondition for marriage and $97.2 \%$ agreed to have a VCT service. The findings of the study indicate that the prevalence of HIV infection is low among high school students in Gondar. The students had adequate knowledge about HIV/AIDS and VCT despite the risky practices. Continued health education is needed to bring behavioral changes. [Ethiop.J.Health Dev. 2007;21(2):179-182]
\end{abstract}

\section{Introduction}

Without a cure now its third decade, acquired immunodeficiency syndrome (AIDS) is a cause of serious public health concern in the world. Estimates indicated that over 40 million people world wide are infected with human immunodeficiency virus (HIV), the cause of AIDS (1). More than half of all new HIV infections occur in 15 to 24 year-olds (1). Members of this age group feel less susceptible to adverse outcomes associated with risk behaviors and are therefore at greater risk for HIV/AIDS. Furthermore, high-risk behaviors established in youth often extend into adulthood, making intervention at a younger age imperative to prevent chronic risk behaviors.

In Ethiopia HIV/AIDS is a major public health problem $(1,2)$. An estimated total of over 1.5 million adults and children were infected and living with the virus as of the end of the year 2003 (2). In several other countries, the magnitude of the problem is very high among young adults associated with risky behaviors. The government of Ethiopia has developed a national policy on HIV/AIDS which is designed to guide the implementation of successful programs to prevent spread of the infection (3).
Studies geared that determining the magnitude of HIV infection and assessing behavioral risk factors of its transmission and prevention among the various segments of the population in Ethiopia are scarce $(2,4)$. In order to plan intervention measures it is important to undergo serologic surveys and studies on people's knowledge of HIV infection, sexual behaviors, perceived risk of infection, and attitude on VCT. Therefore, this study was conducted to shed light on such issues by involving high school students in an urban setup in Gondar, Ethiopia.

\section{Methods}

This cross sectional study was conducted in Gondar city, Northwest Ethiopia in 2004. Students of Fasiledes High School were recruited by stratified sampling. Informed consent was obtained from all participants and the study was ethically approved by the research ethics committee of the University of Gondar.

Data on socio-demographic characteristics, knowledge, attitude and practice (KAP) on HIV/AIDS, VCT and risk factors for acquiring HIV were collected by using a self administered, structured and pre-tested questionnaire.

After pre-test counseling for HIV testing by a specialist counselor, venous blood was collected from each student. Sera were separated by centrifugation and kept at $-20^{\circ} \mathrm{C}$

\footnotetext{
${ }^{1}$ Department of Environmental Health, College of Medicine and Health Sciences, University of Gondar, P O Box, 196, Gondar, Ethiopia E-mail; gashawab@yahoo.com; ${ }^{2}$ Department of Micro-biology and Parasitology, College of Medicine and Health Sciences, University of Gondar, P O Box, 196, Gondar, Ethiopia; ${ }^{3}$ Department of Community Health, College of Medicine and Health Sciences, University of Gondar, University of Gondar, P O Box, 196, Gondar, Ethiopia; ${ }^{4}$ Amhara Regional Health Laboratory, Bahirdar, Ethiopia; ${ }^{5}$ Department of Biology, Faculty of Applied Sciences, University of Gondar, P O Box, 196, Gondar, Ethiopia; ${ }^{6}$ Department of Biochemistry, College of Medicine and Health Sciences, University of Gondar, University of Gondar, P O Box, 196, Gondar, Ethiopia
} 
until used. To assure confidentiality of test results, only code numbers were used to identify sera of students. The presence of HIV-1 antibodies was tested using rapid HIV-1 diagnostic test kits following the manufacturers' instructions. The results were interpreted following the current national algorithm for screening of sera for HIV1 infection which was adopted from WHO (5). The data were entered and analyzed using SPSS version 10 software packages.

\section{Results}

Five hundred sixty five high school students were included in the study. Three hundred eleven (55\%) were males and 254 (45\%) were females. Their mean age was $17.1 \pm 1.7$ years, with significant difference between males and females, $17.6 \pm 1.9$ and $16.5 \pm 1.2$ years, respectively, $\mathrm{P}<0.001$. The lowest age for both sexes was 15 years, while the highest ages were 21 years for females and 24 years for males. The majority (97.6\%) were in the age range of 15 to 20 years. Most of the students were Christians (91.3\%) and single (97.7\%).

Only six students were found to be infected with HIV putting its seroprevalence at $1.1 \%$. Half of them responded that they did not practice sex.The majority (97.5\%) of the participants responded that HIV is an etiologic agent for AIDS. Unprotected sex, unsafe blood transfusion, contaminated needles and mother to child transmissions were reported by $84.6 \%, 64.2 \%, 78.8 \%$, and $69 \%$ of the students as the common ways of HIV transmission. Only 3.6\% reported mosquito bite (2.5\%), shaking hands $(0.7 \%)$ and eating and drinking with infected individuals (0.4\%) as mode of HIV transmission. Abstinence, faithfulness to one's partner and use of condom as means to prevent transmission of HIV was responded by $84.1 \%, 60.4 \%$ and $41.8 \%$ of the students, respectively. Avoiding social life with AIDS patients was reported by $1.8 \%$ of the respondents as a way to prevent transmission of HIV.

Eighty eight point one percent of the respondents were agreed that sexual intercourse should not be committed before marriage. A large number (82.5\%) required screening for HIV as a prerequisite for marriage. Ninety seven point two percent volunteered to be tested for HIV if requested and $89.9 \%$ agreed for post test counselling. Of all the respondents, $84(14.9 \%)$ reported to have had sexual intercourse at least once in the past. Ten (11.9\%) of the sexually active respondents had sex with commercial sex workers (CSW) in the past six months and $9(10.7 \%)$ had contracted sexually transmitted diseases (STDs). Out of the 84 sexually active respondents, 46 (54.8\%) did not use condoms. From the 38 respondents who used condoms, only 26 (68.4\%) used always while the other 12 (31.5\%) reported that they use condoms only some times. The mean age of sexual commencement was $16.9 \pm 1.4$ years for both sexes. It was $17.0 \pm 1.3$ for boys and $16.4 \pm 1.6$ years for girls.

\section{Discussion}

This Study has attempted to assess the seroprevalence of HIV infection, KAP on HIV/ADS and VCT among high school students in the study area. Although several studies on KAP on HIV/AIDS have been conducted in different parts of Ethiopia among diverse population groups (6-14), except this study no single study had examined HIV seroprevalence in association with KAP of the respondents. However, an earlier study involving elementary school students of age 10-14 years in a rural small town of Amhara region showed HIV prevalence of $1.4 \%(15)$.

Interestingly, the $1.1 \%$ seroprevalence of HIV observed among the participants in the present study is one of the lowest ever reported from any population group from the country $(2,4)$. Although, there is no data on HIV seroprevalence among high school students in the study area , our previous reports from Gondar indicated very high prevalences of HIV ranging from over $50 \%$ in tuberculosis patients (16), and 5\% in rural and elderly cataract patients (20). In view of the high prevalence of HIV observed in the different population groups in the region, the $1.1 \%$ prevalence of HIV observed among high school students in Gondar is very low. However, considering the young ages and the female majority among the seropositives (66.7\%), HIV infection is still a threat to the generation. It is worth mentioning at this point that a $12.2 \%$ HIV seroprevalence was reported among 490 university students in Southwest Ethiopia (21). In that study, the prevalence of HIV was higher among students who chew chat, drink alcohol and smoke cigarette as compared to non-chewer, non-drinker and non-smokers counterparts.

Considerable proportions of the respondents had adequate knowledge about the common ways of transmission and prevention of the infection. This is comparable to studies done elsewhere in the country (7, 11 , and 14). This is an encouraging finding which should further be strengthened by establishing organizing HIV/AIDS Clubs in those areas.

It is interesting to find out that over $70 \%$ of all the students claimed to inform their partners or family if were diagnosed positive for HIV infection due to the fear of stigmatization and social pressure which in turn might have contributed for the circulation of the virus in the community. Such groups could pose a serious threat and should be educated to change their attitude.

The respondents seem to have favorable attitude on prevention of the diseases as majority of them approved screening for HIV as a prerequisite for marriage and had negative attitude to sex before marriage. These findings are comparable to the findings from a community based study conducted in Gambella by the end of the year 2000 (14). 
The mean age of first sexual contract for females and males obtained in the present study (16.4 and 17.0 years) is comparable to the observations reported in several previous studies $(6,9,11$, and 14$)$. The findings that over a fourth of the sexually active respondents had multiple sexual partners including CSWs indicate that such risky behavior can predispose the students to acquisition of STDs including HIV. Furthermore, condoms were used only some times by about a third of those who were practicing sexual intercourse. It is worth noting that only about $42 \%$ of the respondents believed use of condom as a means to prevent transmission of HIV. Inline with this, risky sexual behaviors among college students in association with their condom utilization has recently been reported from Gondar (12).

In summary, the prevalence of HIV infection is low among high school students in Gondar. In addition, eventhough the majority of the students had adequate knowledge about HIV/AIDS and VCT, their perception of risk of acquisition and practice of protected sex is low. These call for continued and strengthened health education to bring behavioral changes among the students.

\section{Acknowledgements}

The study was financially supported by a grant from University of Gondar, Ethiopia. Mr Baye Mekuria and Mr Asmamaw Asres of the Fasiledes High School are thanked for facilitating the study. We would like to thank the study participants without whom this study could not have been completed.

\section{References}

1. Joint United Nation Program on HIV-1/AIDS. UNAIDS/WHO. AIDS epidemic update. 2005. World Health Organization, Geneva, 2005.

2. Ministry of Health. AIDS in Ethiopia. 5th ed. Disease Prevention and Control Department, Ministry of Health, Addis Ababa, Ethiopia, 2004.

3. Government of Ethiopia. Policy on HIV/AIDS of the Federal Democratic Republic of Ethiopia. August, 1998. Addis Ababa, Ethiopia.

4. Kebede D, Aklilu M, Sanders E. The HIV epidemic and the state of its surveillance in Ethiopia. Ethiop Med J, 2000;38(4):283-302.

5. Ethiopian Health and Nutrition Research Institute. Manual for HIV-1 Diagnosis. 2002. Addis Ababa, Ethiopia.

6. Geiger S. Sexual behavior \& knowledge of HIV/AIDS \& other STDs. A survey of senior high school students Ethiop J Health Dev, 1990;4(2):123131.

7. Ismail S, H/Giorgis F, Legsse D, Alemu E, Regassa K, Abdella M, Shibeshi M. Knowledge, attitude and practice on high risk factors pertaining to HIV/AIDS in rural community. Ethiop Med J, 1995;33:1-6.
8. Petros B, Belayneh S, Mekonen Y. AIDS and college students in Addis Ababa. A study of knowledge, attitude and behavior. Ethiop J Health Dev. 1997;11(2):115-123.

9. Ismail $\mathrm{S}$, Bitsuamk $\mathrm{H}$ and Alemu K. High risk behavior for STD/HIV, pregnancies and contraception among high school students in a rural town, North Western Ethiopia. Ethiop J Health Dev, 1997;11(1):29-36.

10. Taffa N. Sexual activity of out-of-school youth and their knowledge and attitude about STDs and HIV/AIDS in Southern Ethiopia. Ethiop J health Dev, 1998;12(1):17-22.

11. Korra A, Haile M. Sexual behaviors \& level of awareness on reproductive health among youths evidence from Harar, Eastern Ethiopia. Ethiop J Health dev, 1999;13(2):107-113.

12. Fitaw Y and Worku A. High risk sexual behavior and pattern of condom utilization of the Gondar College of Medical Sciences students, North Western Ethiopia. Ethiop J Health Dev., 2002;16(3):335-338.

13. Abera Z. Knowledge, attitude and behavior (KAB) on HIV/AIDS/STDs among workers in the informal sector in Addis Ababa. Ethiop $\mathrm{J}$ health Dev, 2003;17:53-62.

14. Negash Y, Gebre B, Benti D, Bejiga M. A community based study on knowledge, attitude and practice (KAP) on HIV/AIDS in Gambella town, Western Ethiopia. Ethiop J Health Dev, 2003;17(3):205-213.

15. Mengistu G, Jones J. Low prevalence of HIV in the window of hope age group in northwest Ethiopia. Ethiop J Health Dev, 2003;17(1):85-87.

16. Kassu A, Mengistu G, Ayele b, Diro E, Mekonnen F, Ketema D, Moges F, Mesfin T, Getachew A, Ergicho B, Elias D, Wondmikun Y, Aseffa A, Ota F. Infection with HIV and Intestinal Parasites in Adult Tuberculosis Patients in a Teaching Hospital, Northwest Ethiopia. Trop Doc., 2006, In press.

17. Moges F, Kassu A, Mengistu G, Adugna S, Andualem B, Nishikawa H, Ota F. Seroprevalence of Helicobacter pylori in dyspeptic patients and its relationship with ABO blood groups in a University Hospital, Northwest Ethiopia. World J Gastroenterol., 2006; 12:1957-1961.

18. Kassu A, Moges F, Mekonnen F, Mengistu G, Abate E, Mekonnen E, Molla K, Zewde T, Aseffa A, Wondmikun Y, Ota F. Seroprevalence of HIV in blood donors in Gondar, Ethiopia, 1995-2002. Trop Doc, 2006;36(2):106-107.

19. Mulu A, Kassu A, Tessema B, Wondmikun Y, Nishikawa T, Ota T. Seroprevalence of syphilis and HIV-1 during pregnancy in a teaching hospital, Northwest Ethiopia. Ethiop J Health Dev., 2006, Submitted. 
20. Kassu A, Mekonnen A, Bekele A, Abseno N, Moges F, Wondmikun Y, Ota F. HIV and syphilis infection among elderly people in Northwest Ethiopia. Jap J Infect Dis, 2004;57:264-267.
21. Tefera B, Challi J, Yoseph M. HIV seroprevalence among students ofJimma University, Southwest Ethiopia. Ethiop J Health Sci, 2004;14:65-74. 